\title{
Automatic Removal of Foreground Occluder From Multi-Focus Images
}

\author{
Atsushi Yamashita, Fumiya Tsurumi, Toru Kaneko and Hajime Asama
}

\begin{abstract}
View occluders influence the image quality of a subject when occluders exist between a camera and a subject. For example, a blurred fence image interrupts a subject when an image of a scene is captured by a camera through a fence. In this paper, we propose an automatic removal method of foreground occluders from images using multiple focusing. Our method automatically detects foreground occluder regions by using two images with and without flashlight. The influence of foreground occluders is estimated and overlapping effects of foreground occluders are removed by using multiple focus images. Experimental results show the effectiveness of the proposed method.
\end{abstract}

\section{INTRODUCTION}

In this paper, we propose an automatic removal method of foreground occluders from multi-focus images.

View occluders influence the image quality of a subject when occluders exist between a camera and a subject. For example, a blurred fence image interrupts a subject when an image of a scene is captured by a camera through a fence (Fig. 1(a)). Regions of the image interrupted by foreground occluders are different from the actual values of the subjects in lightness and color. This is a significant problem when a robot recognizes environments if it is not easy for the robot to move to the other side of the fence. Therefore, it is very important to restore the subject image without foreground occluder overlap.

There are a lot of image interpolation or restoration techniques for damaged and occluded images [1]-[7]. However, some of them can only treat with line-shape scratches [1][3], because they are the techniques for restoring old damaged films. In addition, noise regions are not detected automatically and it is required that human operators indicate the noise regions interactively [4]-[7].

As to the noise detection, there are automatic detection methods of foreground occluders such as fences [8], [9]. However, these methods focus on only string-like occluding objects. It is important for robots to detect arbitrary noise regions that have various shapes.

There are automatic methods that can remove occluded noises without helps of human operators [10], [11]. They are effective for moving particles, but not for stationary foreground occluders.

As to stationary noises, we propose automatic noise removing methods from multiple images by using a stereo

Atsushi Yamashita and Hajime Asama are with Department of Precision Engineering, The University of Tokyo, 7-3-1 Hongo, Bunkyo-ku, Tokyo 113-8656, Japan (e-mail: \{yamashita, asama\}@robot.t.u-tokyo.ac.jp)

Fumiya Tsurumi and Toru Kaneko are with Department of Mechanical Engineering, Shizuoka University, 3-5-1 Johoku, Naka-ku, Hamamatsu-shi, Shizuoka 432-8561, Japan

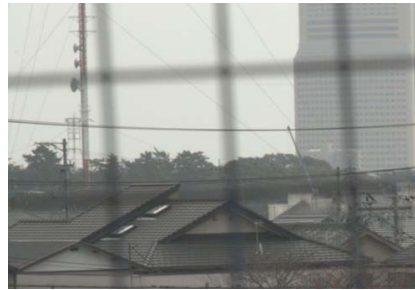

(a) Original image.

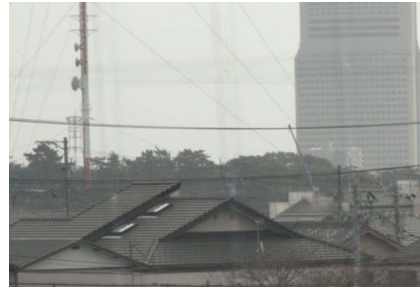

(b) Occluder removal result.
Fig. 1. Example of foreground occluder.

camera [12], [13] and a pan tilt camera [14]-[17]. However, these methods can remove only noises like waterdrops which adhere to the lens protector of the camera.

Automatic noise removal methods by considering blurring effects of foreground occluders are also proposed [18]-[25]. For example, in [22], foreground occluders like fences can be removed from multiple input images with different apertures. This method uses three apertures and estimates the depth of the occluding layer to remove the foreground occluders under the assumption that the occluders have a uniform brightness (single color). However, foreground occluders sometimes have multiple colors.

We propose an automatic removal method of fences that have multiple colors [25] (Fig. 1(b)). When an image of a scene is captured by a camera through a fence, a blurred fence image interrupt objects in the scene, depending on the distance between the camera and the fence. This method can remove the overlapping effects of defocused fence, however, it manually determines the essential parameter of "blurring radius" by trial and error. Theoretically, the blurring radius is calculated from the distance between the camera and the foreground occluder, and the distance between the camera and the background scene. Generally speaking, however, these distances are unknown parameters. In addition, only fence is removed in [25].

In this paper, we proposes a method for determining the blurring radius, and an automatic removing method of foreground occluders from the captured scene using multifocus images regardless of its color and shape. It removes foreground occluders not only fence but also other objects. The method takes advantage of a property that the regions where the foreground occluders exist do not completely lose the information of the background scene.

\section{Removal Method of Foregroud OcCluders}

\section{A. Image Acquisition}

The proposed method uses three images. One is an image capturing the scene in focus through defocused foreground 


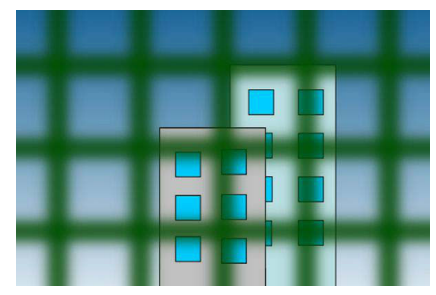

(a) Object focusing image.

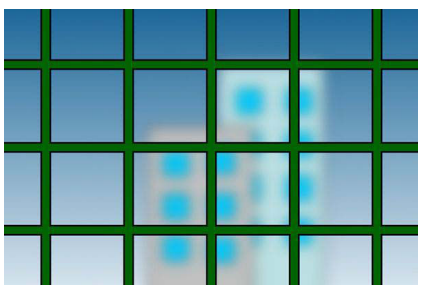

(b) Occluder focusing image without flashlight.

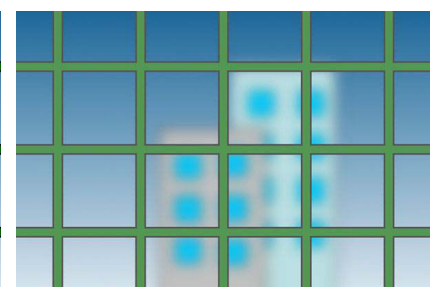

(c) Occluder focusing image with flashlight.

Fig. 2. Image acquisition. Three images are captured, one is an image capturing the scene in focus through the defocused foreground occluder, and the other two are those capturing the foreground occluder in focus.

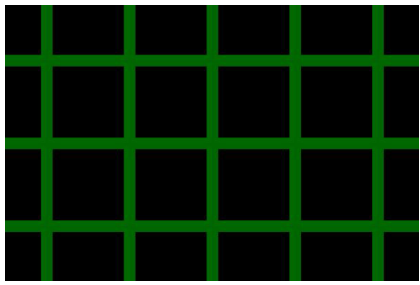

(a) Extraction of occluder.

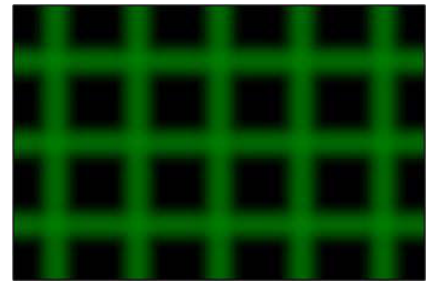

(b) Blur of occluder.
Fig. 3. Extraction and influence of foreground occluders.

occluders (Fig. 2(a)). The other two image are those capturing the foreground occluder in focus (Fig. 2(b)(c)). One of the latter two images is an image with flashlight (Fig. 2(b)) and the other is without flashlight (Fig. 2(c)).

Foreground occluders are removed from the image capturing the scene in focus. The purpose of acquiring two images of the occluder in focus is to extract the occluder regions from the image automatically. Automatic extraction can be realized by subtracting the image without flashlight from the image with flashlight. Distant scene are not influenced by flashlight, while foreground occluders are influenced by flashlight. The difference of pixel value is large in foreground occluder regions and it is small in other regions. Therefore, regions of foreground occluders are extracted by a simple binarization method (Fig. 3(a)).

\section{B. Image Registration}

The registration of the multi-focus images is necessary (Fig. 4) because the scale of the objects in the images has changed with variation of the image distance.

First, the geometrical distortion of the images is corrected by [26]. After correcting the distortion, it is reasonable to suppose that the variation of images is regarded as a scale transform with a magnification factor [27]. This variation of images is expressed with homogeneous coordinates. Let

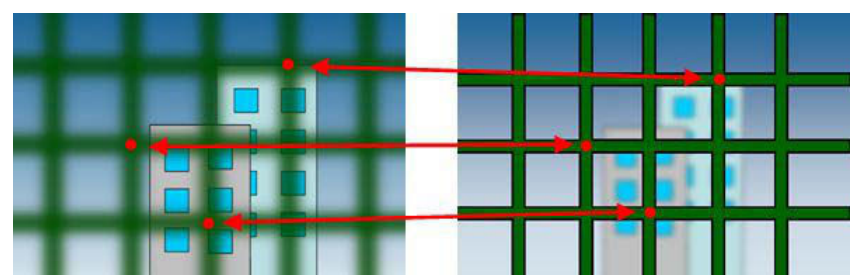

Fig. 4. Image registration. The multi-focus images are registrated because the scale of the objects in the images has changed with variation of the image distance.

$\tilde{\mathbf{x}}_{F}=(u, v, 1)^{T}$ be the homogeneous coordinates of the background subject image, and $\tilde{\mathbf{x}}_{N}=\left(u^{\prime}, v^{\prime}, 1\right)^{T}$ be the homogeneous coordinates of the foreground occluder image. The homogrphy matrix $\mathbf{H}$ gives the magnification and translation from $\tilde{\mathbf{x}}_{F}$ to $\tilde{\mathbf{x}}_{N}$.

$$
\tilde{\mathbf{x}}_{N}=\mathbf{H} \tilde{\mathbf{x}}_{F} .
$$

The least square method is employed to estimate the components of the homogrphy matrix $\mathbf{H}$ to reduce the influence of images noises.

After the homogrphy matrix $\mathbf{H}$ is obtained, the image registration can be executed by using Eq. (1).

\section{Occlusion Model}

Fundamental characteristics of focusing based on the geometrical optics is explained by the thin lens formula under the simple camera model consisting of a thin lens and an image plane.

If the distances from the object to the lens and from the lens to the image are $a$ and $b$ respectively, $a$ and $b$ satisfy the following formula.

$$
\frac{1}{a}+\frac{1}{b}=\frac{1}{f}
$$

where $f$ is the focal length of the lens.

Figure 5(a) shows the geometrical relation between the background objects, the foreground occluders, and the image plane. The background objects are located at distance $a_{F}$ in front of the lens, and the image plane is located at distance $b_{F}$ behind the lens. The foreground occluders are located at distance $a_{N}$, which is shorter than $a_{F}$.

When the background objects are in focus, the foreground occluders are blurred in the image plane (Fig. 3(b)). A point source on the foreground occluders spreads on the image plane as a circle. We call the radius of the circle "blurring radius". The blurring radius $r$ is expressed as follows:

$$
r=\frac{a_{F}-a_{N}}{2 a_{F}} d
$$

where $d$ is the lens radius.

The influence of the foreground occluders can be estimated like PSF (point spread function) if the blurring radius $r$ is known.

Parameter $d$ can be obtained because the lens radius can be measured, however, it is not easy to obtain $a_{F}$ and $a_{N}$ because the positions of the background objects and 


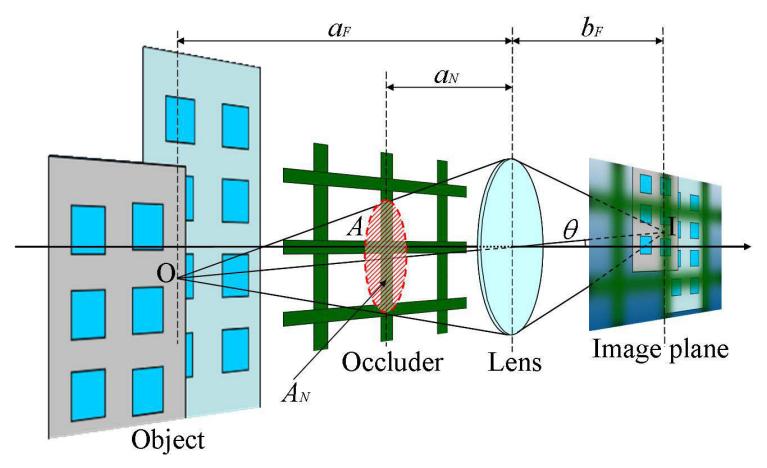

(a) Relation between the background objects, the foreground occluders, and the image plane.

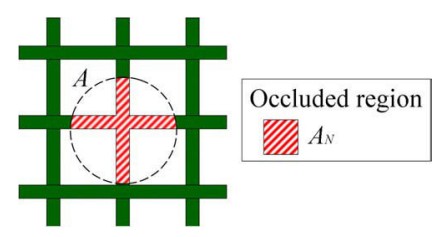

(b) The flux passing region.

Fig. 5. The image is captured when the background objects are in focus.

the foreground occluders are usually unknown when these images are acquired. Therefore, the blurring radius $r$ is estimated only from the images (Section II-D).

In Fig. 5(a), $I$ is a pixel on the object on the image plane and $O$ is the point on the object corresponding to the pixel. The luminous flux radiated from $O$ is focused at $I$ in the image plane by the lens. However, a part of the flux is interrupted by the foreground occluders.

Let $A$ be the area of the flux passing region, and $A_{N}$ be the area of the region interrupted by the foreground occluders, respectively (Fig. 5(b)). Also let $L_{F}$ be the radiance value of $O, E_{F}$ be the irradiance value of $I$, and $L_{N}$ be the radiance value of the region on the foreground occluders, respectively. Then, the relation between the incident radiance value to the lens and irradiance value $E_{F}$ is expressed as follows.

$$
\begin{aligned}
E_{F} & =\frac{\pi d^{2} \cos ^{4} \theta}{b_{F}}\left\{\alpha \int_{A_{N}} \frac{L_{N}}{A_{N}} \cdot d A_{N}+(1-\alpha) L_{F}\right\} \\
& =\alpha k \int_{A_{N}} \frac{L_{N}}{A_{N}} \cdot d A_{N}+(1-\alpha) k L_{F}
\end{aligned}
$$

where $b_{F}$ is the distance between the lens and the image plane, $\theta$ is the angle from the optical center to $I$, and $\alpha$ is a ratio of $A_{N}$ to $A$, respectively.

If the foreground occluder does not exist, Eq. (4) becomes as follows.

$$
E_{F}=k L_{F} .
$$

This means that $k L_{F}$ in Eq. (4) is equivalent to the irradiance value $E_{F}$ which is not interrupted by the foreground occluder. Then, Eq. (4) can be rewritten as follows.

$$
E_{e}=\frac{1}{1-\alpha} E_{F}-\frac{\alpha}{1-\alpha} k \int_{A_{N}} \frac{L_{N}}{A_{N}} \cdot d A_{N},
$$

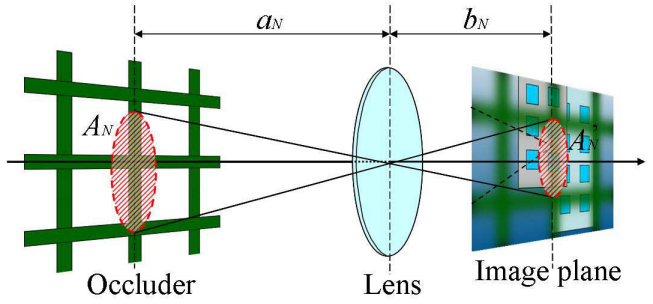

Fig. 6. Relation between the foreground occluders and the image plane when the foreground occluders are in focus.

where $E_{e}=k L_{F}$.

The required parameters to estimate irradiance value $E_{e}$ are the irradiance value $E_{F}$, the radiance value $L_{N}$ and the area of the reversely projected region. In Eq. (6), it is impossible to acquire the radiance value $L_{N}$ directly. Therefore, we use the focused image of the occluder to make Eq. (6) available (Fig. 6). The relation between irradiance value $E_{N}$ and radiance value $L_{N}$ is expressed as follows:

$$
E_{N}=k^{\prime} L_{N} .
$$

Area $A_{N}^{\prime}$ corresponding to $A_{N}$ in Fig. 6 is given as follows.

$$
A_{N}^{\prime}=\frac{b_{N}^{2}}{a_{N}^{2}} A_{N},
$$

where $b_{N}$ is the image distance which is obtained from Eq. (2) with $a=a_{N}$.

Here, let $m=\frac{k}{k^{\prime}}$ be a modification coefficient and $\overline{E_{N}}$ be the average of the irradiance value in $A_{N}^{\prime}$ as follows, respectively.

$$
\overline{E_{N}}=\int_{A_{N}^{\prime}} \frac{E_{N}}{A_{N}^{\prime}} \cdot d A_{N}^{\prime} .
$$

Finally, $E_{e}$ can be obtained by substituting Eqs. (7) and (8) to Eq. (6).

$$
E_{e}=\frac{1}{1-\alpha} E_{F}-m \frac{\alpha}{1-\alpha} \overline{E_{N}} .
$$

If the blurring radius $r$ is known, the value $\alpha$ is acquired by calculating the area of the foreground occluder included in the region. The value $\overline{E_{N}}$ is also acquired by averaging the pixel values of the foreground occluders included in the region. The value $E_{F}$ is obtained from the background scene focused image. Then, the value $E_{e}$ can be acquired by Eq. (10), and the occluder-free image can be generated from the value $E_{e}$.

\section{Estimation of Blurring Radius}

The change of pixel value (brightness) is utilized to estimate the blurring radius.

Figure 7 shows the removal results of the foreground occluders from Fig. 1(a). Figure 7(a) shows the removal result under the assumption of $r=15$, Fig. 7(b) shows the result under the assumption of $r=22$, and Fig. 7(c) shows the ground truth, respectively. Figure 7(c) is acquired by removing the foreground occluder (fence) physically. 


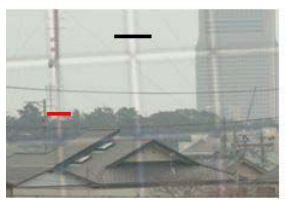

(a) $r=15$.

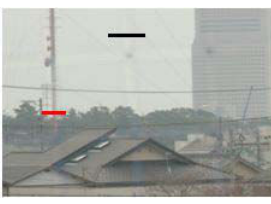

(b) $r=22$.

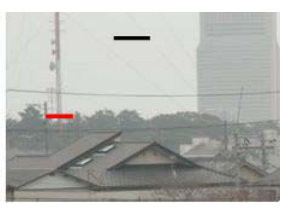

(c) Ground truth.
Fig. 7. Removal results of foreground occluders from Fig. 1(a).

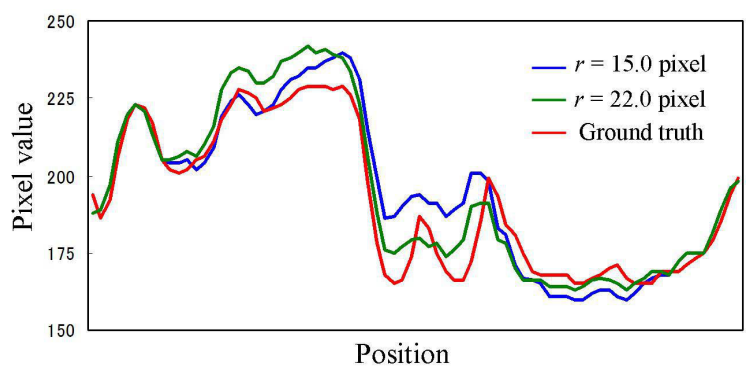

(a) Red line.

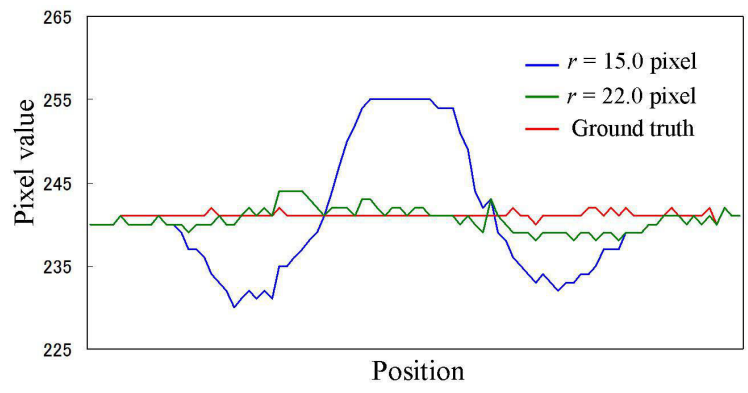

(b) Black line.

Fig. 8. The change of pixel value.

Occluder removal is not good in Fig. 7(a), and is good in Fig. 7(b).

Figures 8(a) and (b) show the pixel value on the red and black lines in Fig. 7, respectively.

The background scene on the red line in Fig. 7 is not simple and has a complicated texture. Therefore, the profile (the graph of pixel value) on the red line changes and is not constant in the ground truth image (red line in Fig. 8(a)). In other words, the variation of pixel value in the ground truth image is large (red line in Fig. 8(a)). The variation of the occluder removal result when $r$ is set as 15 (blue line in Fig. 8(a)) and that when $r=22$ (green line in Fig. 8(a)) are large, too. The profiles on the red line when $r=15$, $r=22$, and the ground truth are difficult to distinguish with each other.

On the other hand, the background scene on the black line in Fig. 7 is not complicated and the variation is small. The variation of pixel value in the ground truth image (red line in Fig. 8(b)) and that when $r=22$ (green line in Fig. 8(b)) are small, while the variation when $r=15$ (blue line in Fig. 8(b)) is large.

To sum up, the variation when the estimated blurring radius $r$ is correct becomes small on simple background regions. Therefore, the regions where the variance of pixel

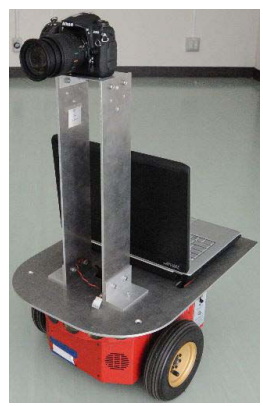

(a) Camera.

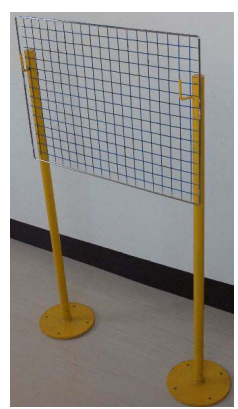

(b) Foreground occluder (fence).
Fig. 9. Experimental setup

value is small are detected, and then the optimal blurring radius when the variance of pixel value in these region becomes the smallest is calculated.

Background regions whose textures are simple have small variance of pixel value. The variance $\sigma$ is calculated as follows:

$$
\sigma^{2}=\frac{1}{n^{2}} \sum_{j=1}^{n} \sum_{i=1}^{n}(x(i, j)-\bar{x})^{2},
$$

where $n$ is the block size of the region of interest, $x(i, j)$ is the pixel value at pixel $(i, j)$, and $\bar{x}$ is the pixel value average of $n \times n$ pixels, respectively.

The estimation (optimization) of $r$ is executed in the region where the variance $\sigma$ is smaller than a threshold value as follows.

$$
f(r)=\frac{1}{n^{2}} \sum_{k=1}^{m} \sum_{j=1}^{n} \sum_{i=1}^{n}\left(x^{\prime}(i, j)-\overline{x^{\prime}}\right)^{2} \rightarrow \min ,
$$

where $m$ is the number of the pixel in the small texture regions.

\section{EXPERIMENT}

We performed experiments in indoor and outdoor environments. Images were acquired through several foreground occluders.

Figure 9 shows one of the experimental setups. Digital camera Nikon D700 was equipped with a mobile robot (Fig. 9(a)) and images were taken through the fence (Fig. 9(b)) in an indoor environment.

Figure 10(a) shows the image taken through the foreground occluder (fence). A person in the image are interrupted by the blurred fence. Figures 10(b) and (c) show the images of the fence in focus without and with flashlight, respectively. Note that the person was moving between the object focusing image and the occluder focusing images were acquired. It is not necessary to take images at the same time. Figure 10(d) shows the removal result of the foreground occluder.

Figure 11 shows the removal result of another foreground occluder. In this case, the foreground occluder was a signboard whose colors are blue and yellow.

Figure 12 shows the experimental result in the outdoor environment. Figure 12(a) shows a fence removal result by 


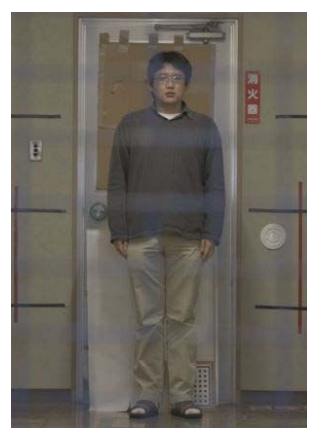

(a) Object focusing image.

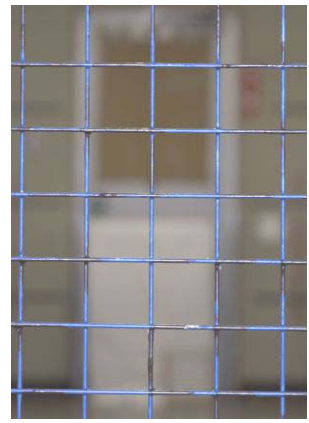

(c) Image with flashlight.

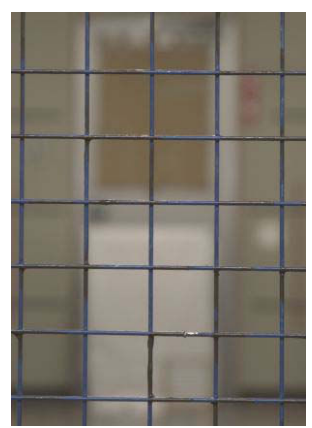

(b) Occluder focusing image.

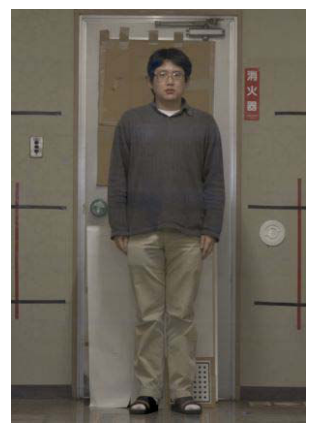

(d) Occluder removal image.
Fig. 10. Experimental result I (Indoor environment).

our proposed method, and Fig. 12(c) shows a fence removal result by image inpainting method [4] for comparison. Both resultant images are converted into grayscale images to compare with an image in which the fence is removed physically. Figure 12(b) is the difference image of Fig. 12(a) and the ground truth image. Figure 12(d) shows the difference image of the reconstruction image by image inpainting method (Fig. 12(c)) and the ground truth. The difference result of image inpainting method has regions with large different values comparing with our method.

Figures 13(a), (b), and (c) show an original image that was taken in outdoor environments, the automatic extraction result of foreground occluders, and the region where $r$ is estimated by using Eq. (11). Figure 14 shows the removal results of the foreground occluders when the blurring radius $r$ changes from 15 to 40 . The influence of the foreground occluders changes while $r$ changes. The influence of the foreground occluders is excessively removed from the original image when $r$ is small (e.g. Fig. 14(a)), and that is undervalued when $r$ is large (e.g. Fig. 14(f)).

The optimal value of $r$ is estimated as 22.3pixel by using Eq. (12) (Fig. 15(a)). Figures 15(b) and (c) show the calculation results of RMSE (root mean squared error) between the original image (Fig. 13(a)) and the ground truth image, and between the result image (Fig. 15(a)) and the ground truth image, respectively. Red color means large error and blue color means small error.

From this result, it is verified that the value of RMSE is reduced drastically in our result.

Figures 16, 17, and 18 show another results. The texture of the background scene in Fig. 16(a) is much

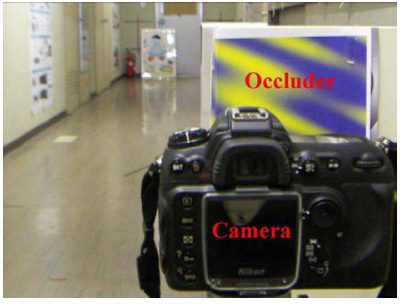

(a) Overview.

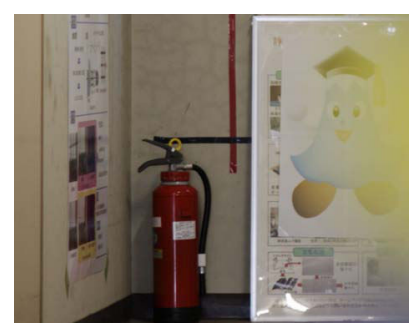

(b) Original image.

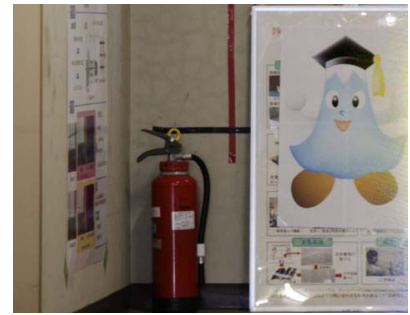

(c) Occluder removal image.

Fig. 11. Experimental result II (Indoor environment).

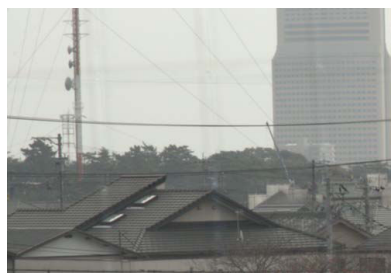

(a) Our result.

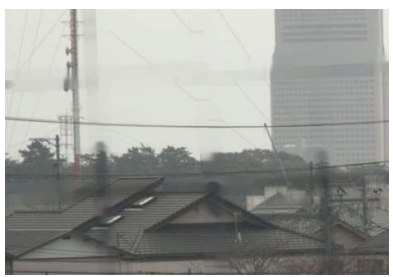

(c) Inpainting result.

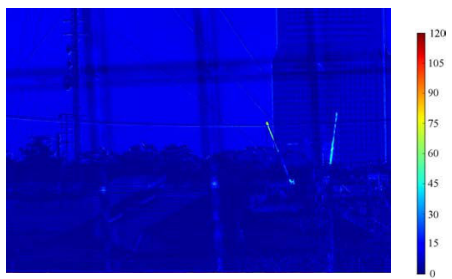

(b) Quantitative evaluation (a).

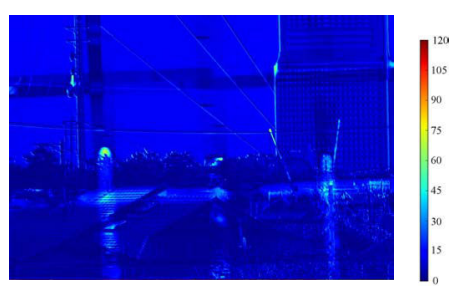

(d) Quantitative evaluation (c).
Fig. 12. Experimental result III (Outdoor environment).

more complicated than that of Fig. 13(a), and the region where $r$ is estimated by using Eq. (11) is small (Fig. 16(c)) when comparing with Fig. 13(c). The optimal value of $r$ is estimated as 29.4pixel in this case (Fig. 18(a)).

From this result, it is verified that our method can treat with several background scenes by estimating the blurring radius automatically.

\section{CONCLUSiON}

In this paper, we propose an automatic removal method of foreground occluders from multi-focus images.

We perform the lightness and color adjustment based on the relation between pixels and the radiance value of the background scenes and the foreground occluders. The influence of blurring effects of foreground occluders is considered automatically by estimating the blurring radius. As a result, defocused foreground occluders are eliminated from the original image. 


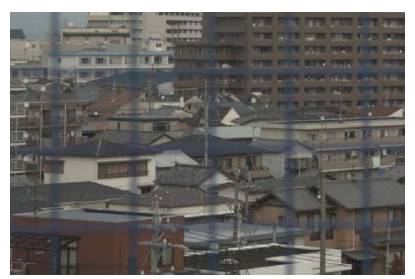

(a) Original image.

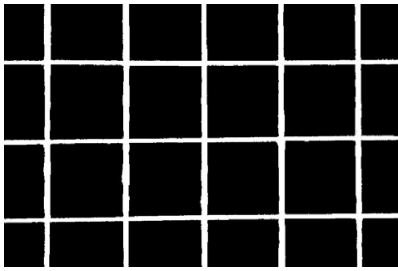

(b) Extracted occluder region

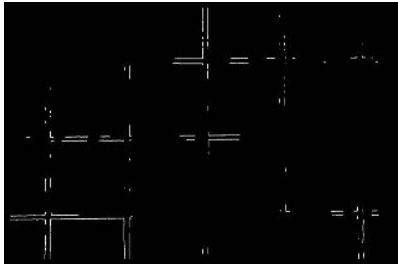

(c) Region where $r$ is estimated.

Fig. 13. Experiment IV (Outdoor environment).

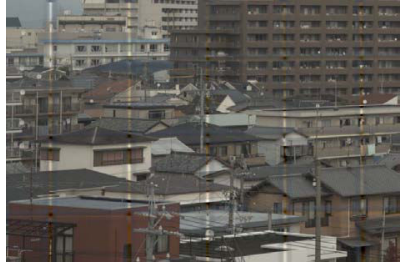

(a) $r=15$.

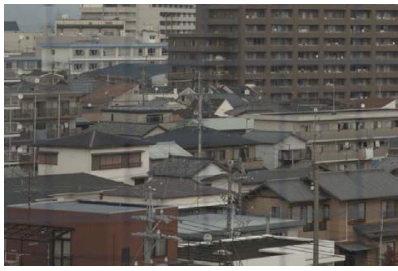

(c) $r=25$

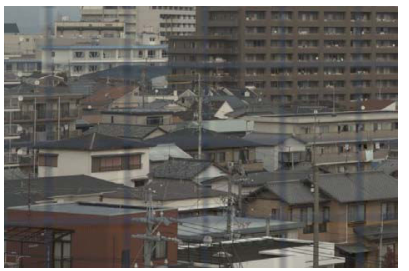

(e) $r=35$

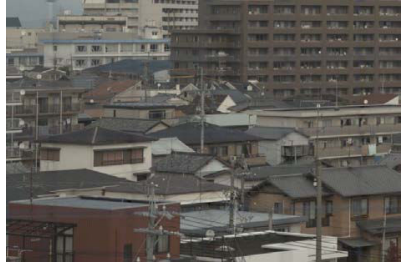

(b) $r=20$.

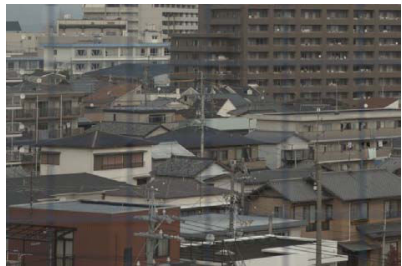

(d) $r=30$

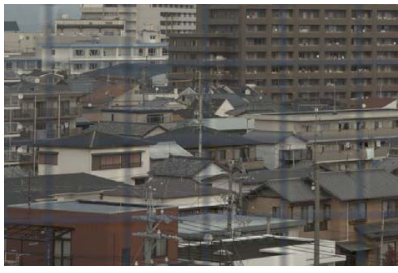

(f) $r=40$.

Fig. 14. The Influence of blurring radius in experiment IV

Experimental results show the effectiveness of the proposed method.

As future works, images have to be acquired in more simple ways. Two images without and with flashlight can be taken at the same time by using a commercially available camera such as FUJI FILM FinePix. Multi-focus images can be taken by using a special hardware such as [28].

It is also interesting to use motion of a mobile robot to remove the near occluder influence [17].

\section{ACKNOWLEDGMENT}

This work was in part supported by MEXT KAKENHI, Grant-in-Aid for Young Scientist (A), 22680017.

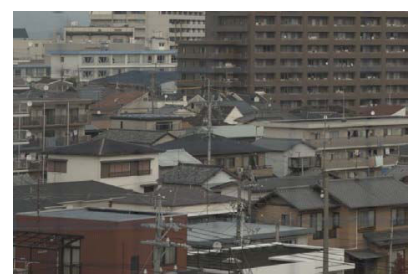

(a) $r=22.3$.

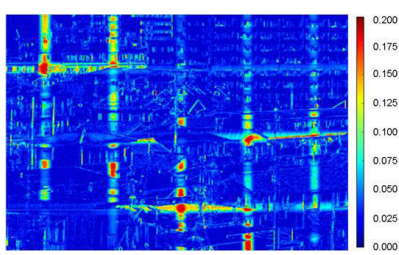

(b) RMSE of original image.

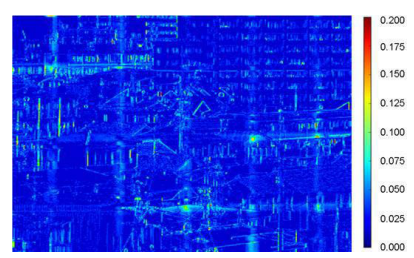

(c) RMSE of result image.
Fig. 15. Experimental result IV.

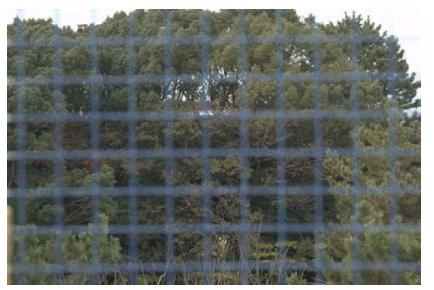

(a) Original image.

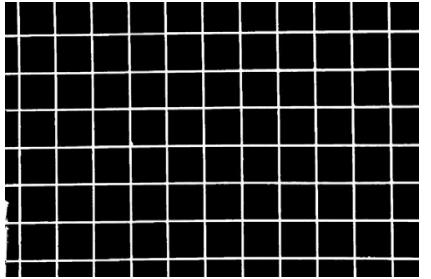

(b) Extracted occluder region.

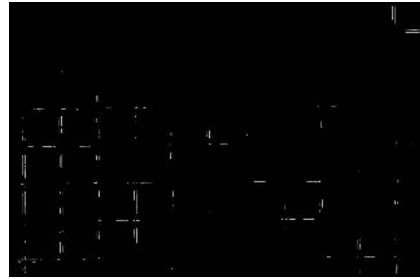

(c) Region where $r$ is estimated.

Fig. 16. Experiment V (Outdoor environment)

\section{REFERENCES}

[1] Anil C. Kokaram, Robin D. Morris, William J. Fitzgerald and Peter J. W. Rayner: "Interpolation of Missing Data in Image Sequences," IEEE Transactions on Image Processing, Vol.4, No.11, pp.1509-1519, 1995.

[2] Simon Masnou and Jean-Michel Morel: "Level Lines Based Disocclusion," Proceedings of the 1998 IEEE International Conference on Image Processing (ICIP1998), pp.259-263, 1998.

[3] Laurent Joyeux, Olivier Buisson, Bernard Besserer and Samia Boukir: "Detection and Removal of Line Scratches in Motion Picture Films," Proceedings of the 1999 IEEE Computer Society Conference on Computer Vision and Pattern Recognition (CVPR1999), pp.548-553, 1999.

[4] Marcelo Bertalmio, Guillermo Sapiro, Vincent Caselles and Coloma Ballester: "Image Inpainting," Proceedings of the 27th Annual Conference on Computer Graphics and Interactive Techniques (SIGGRAPH2000), pp.417-424, 2000.

[5] Yasuyuki Matsushita, Eyal Ofek, Xiaoou Tang and Heung-Yeung Shum: "Full-frame Video Stabilization," Proceedings of the 2005 IEEE Computer Society Conference on Computer Vision and Pattern Recognition (CVPR2005), Vol.1, pp.50-57, 2005.

[6] Yuping Shen, Fei Lu, Xiaochun Cao and Hassan Foroosh: "Video Completion for Perspective Camera Under Constrained Motion," Proceedings of the 18th International Conference on Pattern Recognition (ICPR2006), Vol.3, pp.63-66, 2006. 


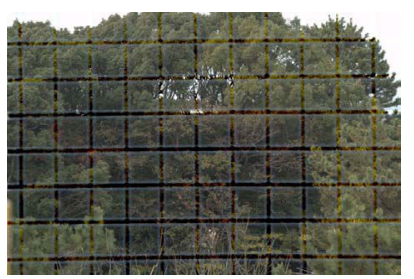

(a) $r=15$.

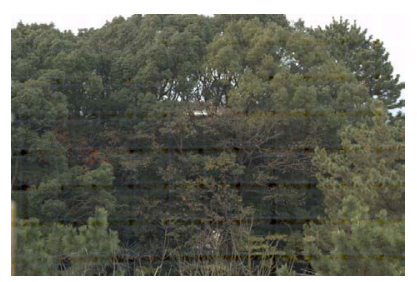

(c) $r=25$.

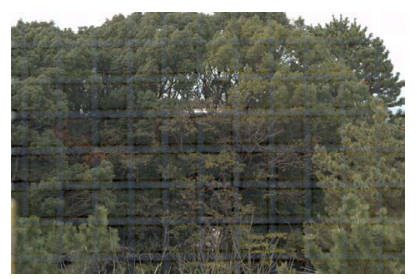

(e) $r=35$.

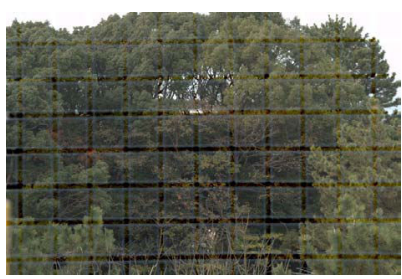

(b) $r=20$.

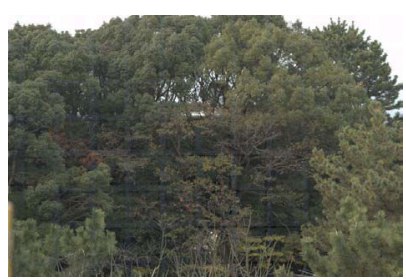

(d) $r=30$.

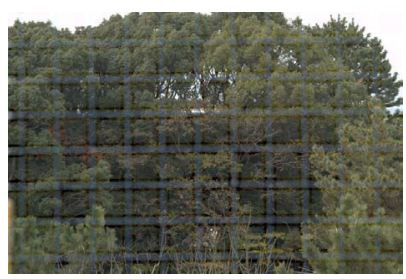

(f) $r=40$.
Fig. 17. The Influence of blurring radius in experiment V.

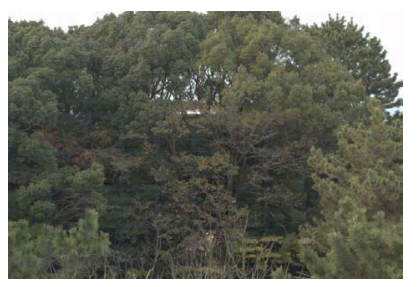

(a) $r=29.4$.

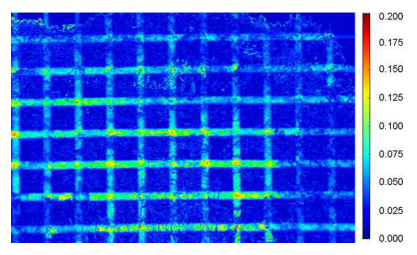

(b) RMSE of original image.

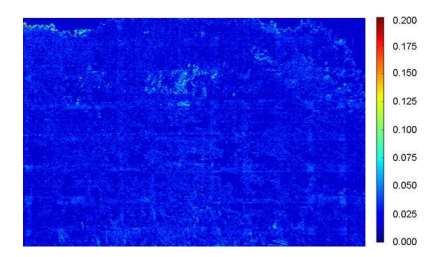

(c) RMSE of result image.
Fig. 18. Experimental result V.

[7] Yonatan Wexler, Eli Shechtman and Michal Irani: "Space-Time Completion of Video," IEEE Transactions on Pattern Analysis and Machine Intelligence, Vol.29, No.3, pp.463-476, 2007.

[8] Toru Tamaki, Hiroshi Suzuki and Masanobu Yamamoto: "String-like Occluding Region Extraction for Background Restoration," Proceedings of the 18th International Conference on Pattern Recognition (ICPR2006), Vol.3, pp.615-618, 2006.

[9] Minwoo Park, Kyle Brocklehurst, Robert T. Collins and Yanxi Liu: "Deformed Lattice Detection in Real-World Images Using MeanShift Belief Propagation," IEEE Transactions on Pattern Analysis and Machine Intelligence, Vol.31, No.10, pp.1804-1816, 2009.

[10] Hiroyuki Hase, Kazunaga Miyake and Masaaki Yoneda: "Real-time Snowfall Noise Elimination," Proceedings of the 1999 IEEE International Conference on Image Processing (ICIP1999), Vol.2, pp.406409, 1999.

[11] Kshitiz Garg and Shree K. Nayar: "Detection and Removal of Rain from Videos," Proceedings of the 2004 IEEE Computer Society
Conference on Computer Vision and Pattern Recognition (CVPR2004), Vol.1, pp.528-535, 2004.

[12] Atsushi Yamashita, Masayuki Kuramoto, Toru Kaneko and Kenjiro T. Miura: "A Virtual Wiper -Restoration of Deteriorated Images by Using Multiple Cameras," Proceedings of the 2003 IEEE/RSJ International Conference on Intelligent Robots and Systems (IROS2003), pp.3126-3131, 2003.

[13] Atsushi Yamashita, Yuu Tanaka and Toru Kaneko: "Removal of Adherent Waterdrops from Images Acquired with Stereo Camera," Proceedings of the 2005 IEEE/RSJ International Conference on Intelligent Robots and Systems (IROS2005), pp.953-958, 2005.

[14] Atsushi Yamashita, Toru Kaneko and Kenjiro T. Miura: "A Virtual Wiper -Restoration of Deteriorated Images by Using a Pan-Tilt Camera-," Proceedings of the 2004 IEEE International Conference on Robotics and Automation (ICRA2004), pp.4724-4729, 2004.

[15] Atsushi Yamashita, Tomoaki Harada, Toru Kaneko and Kenjiro T. Miura: "Removal of Adherent Noises from Images of Dynamic Scenes by Using a Pan-Tilt Camera," Proceedings of the 2004 IEEE/RSJ International Conference on Intelligent Robots and Systems (IROS2004), pp.437-442, 2004.

[16] Atsushi Yamashita, Isao Fukuchi, Toru Kaneko and Kenjiro T. Miura: "Removal of Adherent Noises from Image Sequences by SpatioTemporal Image Processing," Proceedings of the 2008 IEEE International Conference on Robotics and Automation (ICRA2008), pp.23862391, 2008.

[17] Atsushi Yamashita, Isao Fukuchi and Toru Kaneko: "Noises Removal from Image Sequences Acquired with Moving Camera by Estimating Camera Motion from Spatio-Temporal Information," Proceedings of the 2009 IEEE/RSJ International Conference on Intelligent Robots and Systems (IROS2009), pp.3794-3801, 2009.

[18] Naoki Asada, Hisanaga Fujiwara and Takashi Matsuyama: "Seeing Behind the Scene - Analysis of Photometric Properties of Occluding Edges by the Reversed Projection Blurring Model-," IEEE Transactions on Pattern Analysis and Machine Intelligence, Vol.20, No.2, pp.155-167, 1998.

[19] Paolo Favaro and Stefano Soatto: "Seeing Beyond Occlusions (And Other Marvels of A Finite Lens Aperture)," Proceedings of the 2003 IEEE Computer Society Conference on Computer Vision and Pattern Recognition (CVPR2003), Vol.2, pp.579-586, 2003.

[20] Vaibhav Vaish, Richard Szeliskiy, C. L. Zitnicky, Sing Bing Kang and Marc Levoy: "Reconstructing Occluded Surfaces using Synthetic Apertures: Stereo, Focus and Robust Measures," Proceedings of the 2006 IEEE Computer Society Conference on Computer Vision and Pattern Recognition (CVPR2006), Vol.2, pp.2331-2338, 2006.

[21] Yanxi Liu, Tamara Belkina, James H. Hays and Roberto Lublinerman: "Image De-fencing," Proceedings of the 2008 IEEE Computer Society Conference on Computer Vision and Pattern Recognition (CVPR2008), pp.1-8, 2008.

[22] Jinwei Gu, Ravi Ramamoorthi, Peter Belhumeur and Shree Nayar: "Removing Image Artifacts Due to Dirty Camera Lenses and Thin Occluders," ACM Transactions on Graphics (Proceedings of SIGGRAPH ASIA 2009), Vol.28, No.5, 2009.

[23] Scott McCloskey, Michael Langer and Kaleem Siddiqi: "Romoving Partial Occlusion from Blurred Thin Occluders," Proceedings of the 20th International Conference on Pattern Recognition (ICPR2010), pp.4400-4403, 2010.

[24] Minwoo Park, Kyle Brocklehurst, Robert T. Collins and Yanxi Liu: "Image De-Fencing Revisited," Proceedings of the 10th Asian Conference on Computer Vision (ACCV2010), pp.2522-2534, 2010.

[25] Atsushi Yamashita, Akiyoshi Matsui and Toru Kaneko: "Fence Removal from Multi-Focus Images," Proceedings of the 20th International Conference on Pattern Recognition (ICPR2010), pp.4532-4535, 2010.

[26] Juyang Weng, Paul Cohen and Marc Herniou: "Camera Calibration with Distortion Models and Accuracy Evaluation," IEEE Transactions on Pattern Analysis and Machine Intelligence, Vol.14, No.10, pp.965980, 1992.

[27] Reg G. Willson and Steven A. Shafer: "What is the Center of the Image?," Journal of the Optical Society of America, Vol.11, No.11, pp.2946-2955, 1994.

[28] Shinsaku Hiura and Takashi Matsuyama: "Depth Measurement by the Multi-Focus Camera," Proceedings of the 1998 IEEE Computer Society Conference on Computer Vision and Pattern Recognition (CVPR1998), pp.953-959, 1998. 